\title{
Sinusitis maxilar iatrogénica tras la colocación de implantes: A propósito de un caso
}

\section{Iatrogenic maxillary sinusitis related to implant placement: $\boldsymbol{A}$ case report}

\author{
BRAGADO NOVEL M* \\ BERINI AYTÉS L** \\ GAY ESCODA C $* * * *$
}

\begin{abstract}
Bragado Novel M, Berini Aytés L, Gay Escoda C. Sinusitis maxilar iatrogénica tras la colocación de implantes: A propósito de un caso. Av Periodon Implantol. 2010; 22, 3: 135-145
\end{abstract}

\section{RESUMEN}

La perforación del suelo del seno maxilar y de las fosas nasales durante la colocación de implantes en las crestas alveolares situadas por debajo no es un hecho infrecuente. Pequeñas comunicaciones producidas durante el fresado para la preparación del lecho implantario cierran espontáneamente, pero es un requisito indispensable para evitar futuras complicaciones que el implante quede estable, si no deberá ser retirado.

Presentamos el caso de una mujer de 54 años de edad que acude refiriendo cacosmia y halitosis permanentes y episodios esporádicos de dolor en la zona infraorbitaria derecha asociados a rinorrea purulenta, que no ceden a la administración de distintos antibióticos. Las pruebas radiológicas muestran tres implantes dentales en el primer cuadrante sustituyendo a 1.2, 1.3 y 1.6: el primero perforando la fosa nasal, y el tercero con el extremo apical en el interior del seno maxilar derecho con una imagen radioopaca bien delimitada asociada. Confirmada la existencia de una comunicación bucosinusal de más de dos años de evolución, y establecida esta como la causa del cuadro de sinusitis maxilar crónica que la paciente padece, se determina como plan de tratamiento la extracción de los tres implantes y la reconstrucción del plano óseo mediante un injerto de hueso autólogo de mentón. Al retirar el implante en posición 1.6 se observa material de aspecto resinoso adherido a toda su superficie, con fines probablemente retentivos, y que habrá contribuido en gran medida a incrementar el proceso irritativo local. El defecto remanente es tan importante en este sector que se hace imposible anclar un injerto óseo en este área, por lo que se legra la mucosa sinusal enferma a su través, enviando muestra para su posterior análisis anatomopatológico, y se consigue el cierre primario mediante un injerto pediculado de la bola adiposa de Bichat. Los controles postoperatorios fueron favorables consiguiendo de esta forma la remisión total de la sintomatología y la recuperación completa.

PALABRAS CLAVE: Implantes dentarios, comunicación bucosinusal, sinusitis maxilar, colgajo de la bola adiposa de Bichat, iatrogenia.

\section{SUMMMARY}

Perforation of the floor of the maxillary sinus or nasal cavity during implant placement in the residual bone located below is not an infrequent situation. It's been strongly reported that slight membrane puncture while preparing implant bed does not play a significant role in clinical outcome, as it heals spontaneously, but it is an essential requirement that the implant remains intraoperatory stable.

* Odontóloga. Residente del Máster de Cirugía Bucal e Implantología Bucofacial. Facultad de Odontología de la Universidad de Barcelona.

** Profesor Titular de Patología Quirúrgica Bucal y Maxilofacial. Profesor del Máster de Cirugía Bucal e Implantología Bucofacial. Facultad de Odontología de la Universidad de Barcelona. Investigador del Instituto IDIBELL.

*** Catedrático de Patología Quirúrgica Bucal y Maxilofacial. Director del Máster de Cirugía Bucal e Implantología Bucofacial. Facultad de Odontología de la Universidad de Barcelona. Investigador coordinador del Instituto IDIBELL. Jefe del Servicio de Cirugía Bucal, Implantología Bucofacial y Cirugía Maxilofacial del Centro Médico Teknon. Barcelona. 
We present the case of a $54 \mathrm{yr}$. old woman that consulted in our office referring permanent cacosmia and halitosis associated to sporadic episodes of pain and purulent rhinorrhea, that didn't ease to the administration of several different antibiotics. Radiological findings show three dental implants in the first quadrant replacing teeth 1.2, 1.3 and 1.6: first one piercing the nostril and the third one with its apical end completely into the right maxillary sinus with a delimitated radiopaque mass associated. Once confirmed the existency of an oroantral fistula and its involvement in the two-year evolution chronic sinusitis diagnosed, the treatment established included the extraction of the three implants and the simultaneous osseous reconstruction with an autologous bone chin graft.

After the removal of implant 1.6, a composite resin-like material is found stuck to its surface with supposedly retentive purposes, contributing in this way in a very strong way to increase irritation. Remanent defect is so important that makes impossible bone grafting in this area, so we proceed to curette harmed sinusal mucosa, sending a sample for histological evaluation, and close with a Bichat fat pad pedicled flap. Postoperatory follow-up and evolution was favourable, achieving total remission of sympthomatology and complete recovery.

KEY WORDS: Dental implants, oroantral fistula, sinusitis, Bichat fat pad pedicled flap, oatrogenia.

Fecha de recepción: 11 de junio 2009.

Fecha de aceptación: 25 de junio 2009.

\section{INTRODUCCIÓN}

La rehabilitación con implantes de los sectores posteriores del maxilar superior se encuentra con frecuencia comprometida debido a la atrofia sufrida por el hueso alveolar o a la neumatización excesiva de los senos maxilares. Estas limitaciones pueden solventarse realizando, previa la colocación de implantes, técnicas de aumento de la cresta alveolar como son: el injerto "onlay", si la causa de la disminución de altura ósea en este área es una reabsorción severa del proceso alveolar residual; o una elevación del seno maxilar si es el suelo de este el que ha descendido reduciendo la cantidad de hueso disponible. Ambas posibilidades son técnicas con un excelente pronóstico, sin embargo, suponen de forma invariable un aumento del tiempo total de tratamiento y deben efectuarse por profesionales debidamente especializados y entrenados (1).

El hueso del maxilar superior es blando, de baja densidad y calidad, poco trabeculado y con una cortical delgada. Tradicionalmente se considera que la altura mínima de hueso disponible para la colocación de implantes en este área es de $10 \mathrm{~mm}$, y se recomienda el uso de implantes de plataforma ancha. Si un implante colocado en este sector, próximo en su zona apical al suelo del seno maxilar, no presenta intraoperatoriamente una adecuada estabilidad primaria y muestra una movilidad importante, deberá ser retirado, ya que podría existir riesgo de desplazamiento de éste al seno maxilar, y provocar secundariamente una sinusitis maxilar (2).

Existen diversos tratamientos para conseguir el cierre de una comunicación bucosinusal dependiendo de la etiología, la localización, el tamaño y el tiempo transcurrido desde que se produjo. Si existe patología infecciosa sinusal activa es indispensable tratarla previamente a la implementación de cualquier procedimiento quirúrgico (3). La utilización de un colgajo pediculado de la bola adiposa de Bichat para el cierre de comunicaciones oroantrales fue descrito por primera vez por Egyedi (4) en el año 1977; dos avances significativos se han producido modificando la técnica original descrita (5. 6): su utilización como injerto libre para esta misma aplicación, publicada en 1983; y la posibilidad de prescindir del injerto cutáneo para recubrirlo, ya que Tideman y cols. (7) demostraron que el injerto graso no pediculado epitelizaba de forma espontánea en 1 a 4 semanas.

La bola de Bichat es una masa lobulada de tejido adiposo que rellena el espacio delimitado por el masetero, el tendón del músculo temporal y el músculo pterigoideo interno por la parte posterior; el músculo buccinador y su aponeurosis por la parte interna; el primer plano de los músculos cutáneos por la parte externa y finalmente, en la parte anterior, por el conducto de Stensen. En su descripción anatómica se distingue un cuerpo con cuatro prolongaciones: vestibular, superomedial, tem- 
poral y pterigomandibular $(8,9)$. Sus excepcionales características como tejido indicado para la reconstrucción de diferentes estructuras bucofaciales vienen determinadas por su triple irrigación, que procede de las arterias maxilar interna, facial transversa y temporal superficial. Su uso como colgajo pediculado es una técnica ampliamente utilizada para rehabilitar defectos de hasta $6 \mathrm{~cm}$ debido a sus innumerables ventajas (9): es un procedimiento poco invasivo, ofrece una muy baja morbilidad y un excelente postoperatorio, y es sencillo de llevar a cabo por un cirujano experimentado y muy predecible, debido a su reducido porcentaje de complicaciones $(10,11)$.

\section{CASO CLÍNICO}

Mujer de 54 años de edad que acudió en diciembre de 2005 al Servicio de Cirugía Bucal, Implantología Bucofacial y Cirugía Maxilofacial del Centro Médico Teknon remitida por su otorrinolaringólogo. El motivo de consulta era la valoración de una posible sinusitis maxilar derecha de aproximadamente un año de evolución y la asociación de esta con la rehabilitación implantosoportada en el primer cuadrante. Esta hemiarcada fue rehabilitada en febrero de 2001 con cuatro implantes (en posición 1.2, 1.3, 1.5 y 1.6) que fueron retirados 10 meses más tarde debido a una periimplantitis que supuso el fracaso del tratamiento, con una importante pérdida ósea resultante en este sector. Se colocaron en el año 2002 de forma secuencial tres implantes (1.2 en una primera intervención y 1.3 y 1.6 posteriormente) sin la realización de ningún procedimiento de regeneración ósea, que se cargaron protésicamente juntos en 2003 colocando una rehabilitación fija con un póntico en 1.5. La paciente refiere como sintomatología principal cacosmia y halitosis permanentes. El comienzo de los síntomas sugestivos de la existencia de un cuadro sinusal se remonta a 2004. Desde entonces describe diversos episodios agudos de infección en los que las manifestaciones clínicas se hacían más patentes: algias locales y sensación de presión en el maxilar afecto, asociados a la presencia de secreción purulenta nasal y en el esputo, más evidente al levantarse por las mañanas. Estas reagudizaciones remitieron tras la administración de distintos antibióticos, cuyos nombres no recuerda; pero las molestias, de más de un año de evolución, persisten a pesar del tratamiento.

A la exploración intraoral la paciente presenta una periodontitis crónica moderada en los dientes remanentes y un edentulismo parcial con múltiples ausen- cias dentarias: dos prótesis fijas en maxilar superior y una removible articulada con el sector anteroinferior por medio de ataches. La encía adyacente a todos los implantes se muestra tumefacta, eritematosa y sangrante al examen, de forma más marcada en el implante en posición 1.6. El implante en posición 1.3 muestra una pérdida ósea severa y la exposición de sus dos tercios coronales.

En la ortopantomografía realizada en la primera visita (Fig. 1) se aprecia que el implante en posición $1.6 \mathrm{se}$ encuentra casi en su totalidad en el interior del seno maxilar derecho, la base del mismo se desdibuja intuyéndose una gran perforación. El implante en posición 1.3 presenta una pérdida importante de soporte óseo y el 1.2 se insinúa en interior de las fosas nasales.
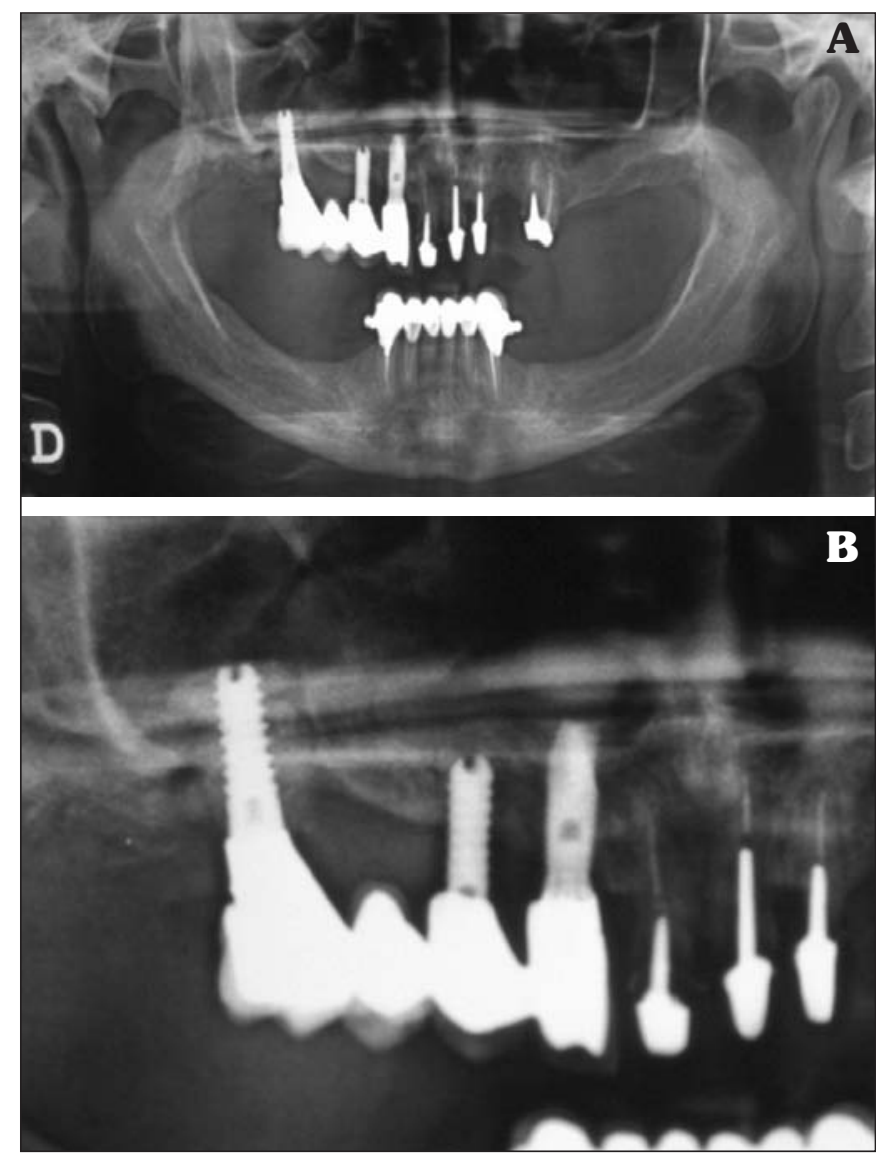

Fig. 1: Ortopantomografía. (A) Valoración del estado bucodental general de la paciente en el momento de nuestra primera visita. La rehabilitación fija en el segundo cuadrante había sido retirada. (B) Detalle del primer cuadrante: Se observa con mayor precisión la rehabilitación implantosoportada 1.2-1.6 que presuntamente ocasionaba la patología: el implante en posición 1.6 perfora el suelo del seno maxilar. 
Se solicita una tomografía computadorizada (TC) para estudiar de forma más exhaustiva el caso y el grado de afectación sinusal, encontrándose una opacificación homogénea en el seno maxilar derecho respecto al contralateral: ocupación de aspecto mucoso-inflamatorio con la presencia de una solución de continuidad en el suelo sinusal. El implante en posición 1.6 se visualiza claramente en el interior del mismo con una imagen radioopaca asociada que en el informe radiológico se traduce como injerto óseo que lo acompaña, aparentemente no integrado (Figs. 2, 3 y 4).
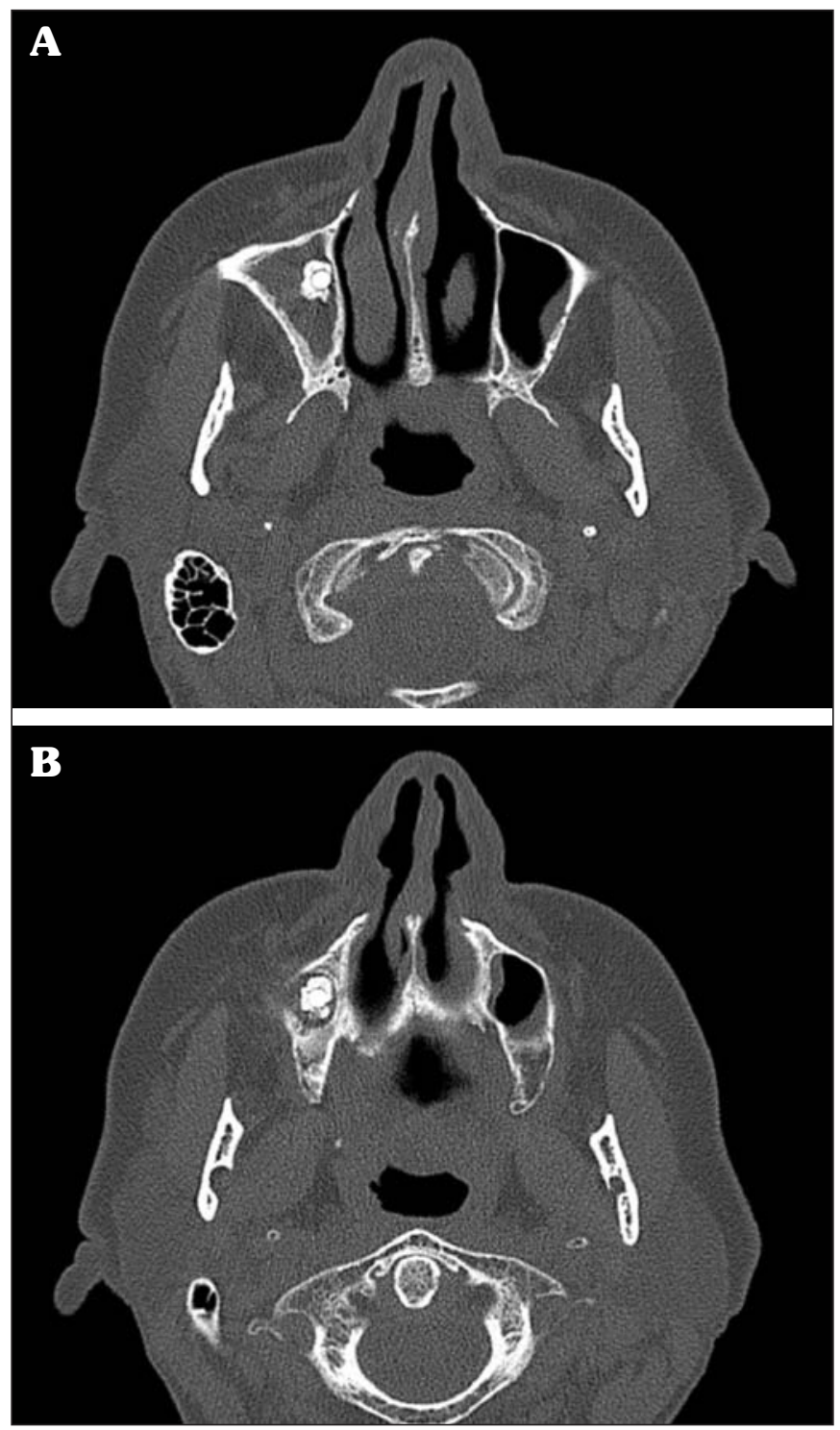

Fig. 2: Secuencia de cortes axiales de la TC en sentido craneo-caudal. (A-B) Imágenes que muestran la comunicación bucosinusal existente a nivel del implante en posición 1.6 y cómo este perfora el suelo del seno maxilar derecho.
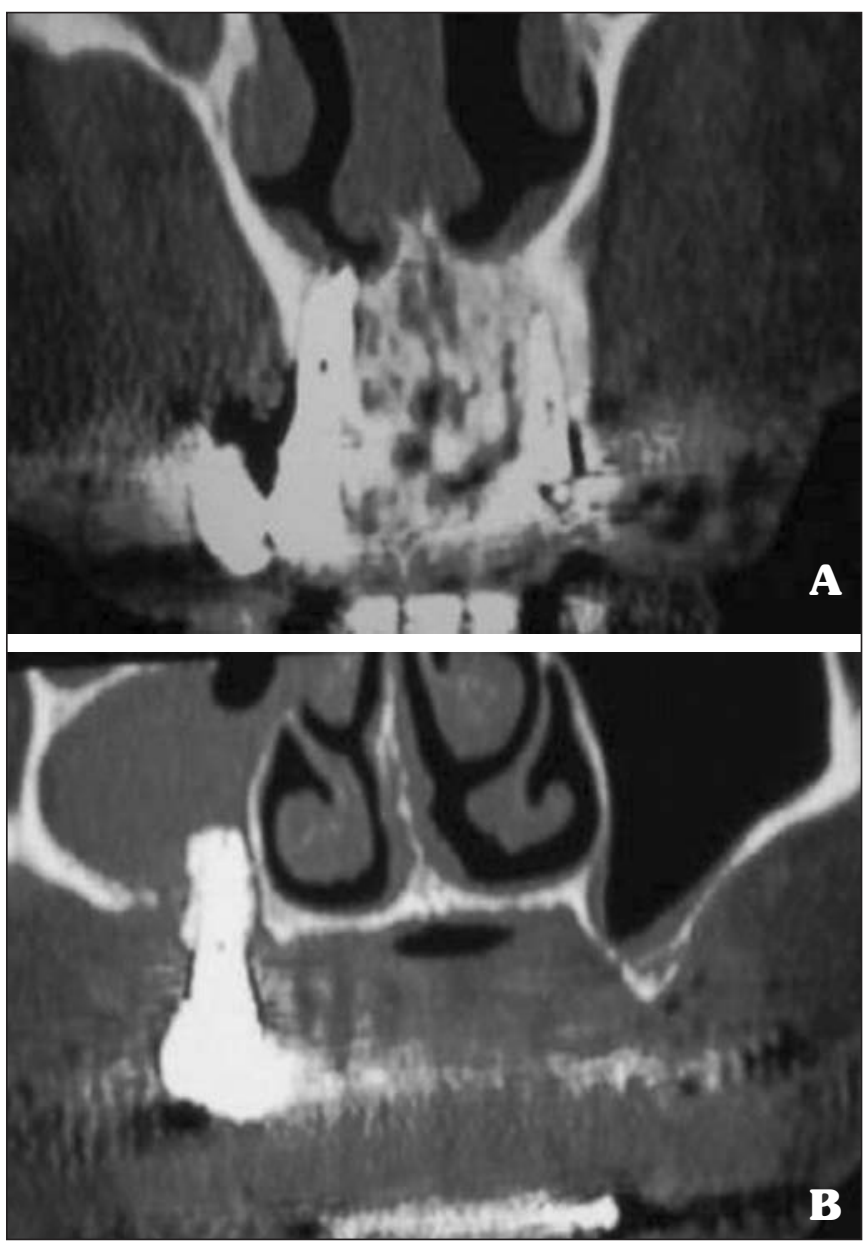

Fig. 3: Secuencia de cortes frontales de la TC en sentido anteroposterior. (A) El implante en posición 1.3 protruye en el suelo de la fosa nasal. (B) El implante en posición 1.6 está casi en su totalidad en el interior del seno maxilar. Se observa una radioopacidad del seno maxilar compatible con hiperplasia de la mucosa sinusal debido a estímulo irritativo prolongado.

Confirmada la existencia de una comunicación bucosinusal de más de dos años de evolución, y establecida esta como la causa del cuadro de sinusitis maxilar crónica que la paciente padece, se establece como plan de tratamiento la extracción de los tres implantes y la reconstrucción del plano óseo mediante un injerto autólogo de hueso del mentón.

Previamente a la fase quirúrgica, se retiran las dos rehabilitaciones fijas superiores ( 1.6 al 1.2 implantosoportada y 1.1 al 2.6 dentosoportada) y se confecciona una prótesis provisional en el segundo cuadrante para restaurar temporalmente los dientes remanentes. El 24 de enero de 2006 se lleva a cabo la intervención quirúrgica bajo anestesia local y sedación consciente por 


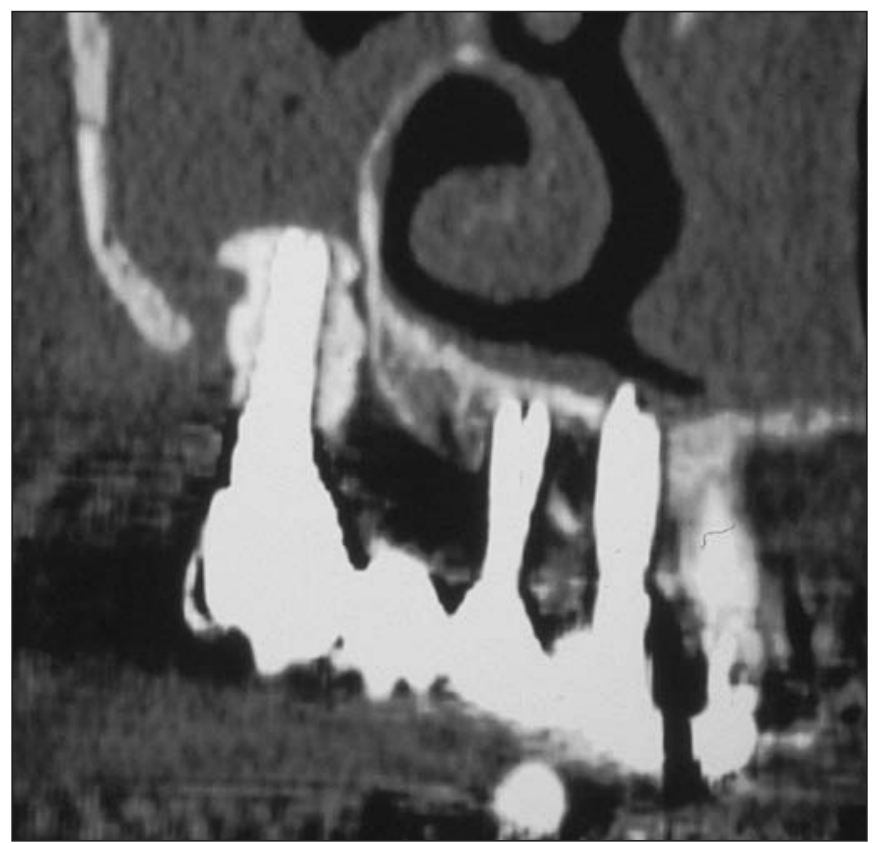

Fig. 4: Detalle de la reconstrucción panorámica de la TC del primer cuadrante. Se visualiza más detalladamente la imagen radioopaca asociada al implante en posición 1.6, la extensión de la comunicación bucosinusal y la patología sinusal existente.

vía endovenosa con midazolam, propofol y remifentanilo. Se efectúa la infiltración de anestesia locorregional paraapical supraperióstica alta en todo el área vestibular del primer cuadrante, reforzando por palatino. El anestésico utilizado fue Articaína 4\% + adrenalina 1:100.000 (Laboratorios Inibsa, Barcelona, España). La incisión horizontal discurre por el sulcus de los implantes afectos, con dos descargas en distal del 1.2 y del implante en posición 1.6, que se prolongaron hacia el fondo del vestíbulo delimitando un colgajo trapezoidal de espesor total y exponiendo de esta forma los tres implantes (Fig. 5A). El implante en posición 1.3 es retirado con facilidad debido a su poco soporte óseo, fue necesario sin embargo utilizar una trefina para extraer el implante en posición 1.2 (Fig. 5B-C). Al retirar el implante situado en posición 1.6 se observa, con sorpresa, la presencia de un material de aspecto resinoso adherido a toda su superficie, con fines probablemente retentivos, y que habrá contribuido en gran medida a incrementar el proceso irritativo y la destrucción ósea circundante (Fig. 6). El defecto óseo creado en el reborde alveolar residual es muy importante (Fig. 7). Se procede a legrar a su través toda la mucosa sinusal patológica enviando una muestra de esta para el posterior análisis anatomopatológico. Se prepara una antrostomía nasal colocando
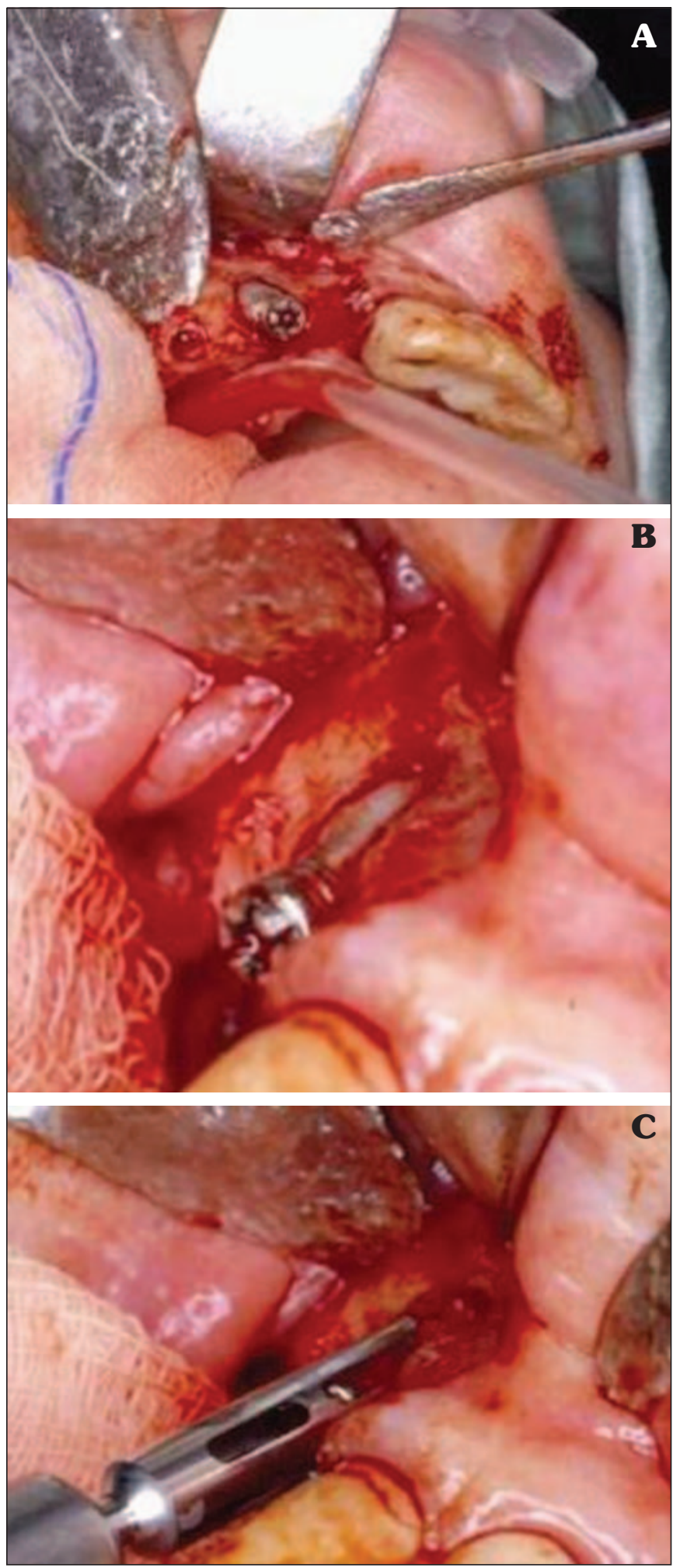

Fig. 5: Secuencia de la extracción de los tres implantes. (A) Aspecto del campo quirúrgico al levantar el colgajo. Implante en posición 1.3 no ofrece resistencia a la extracción. (B) Implante en posición 1.2 se mantiene estable a pesar de reabsorción ósea vestibular severa. (C) Uso de la trefina para retirar el implante en posición 1.2. 

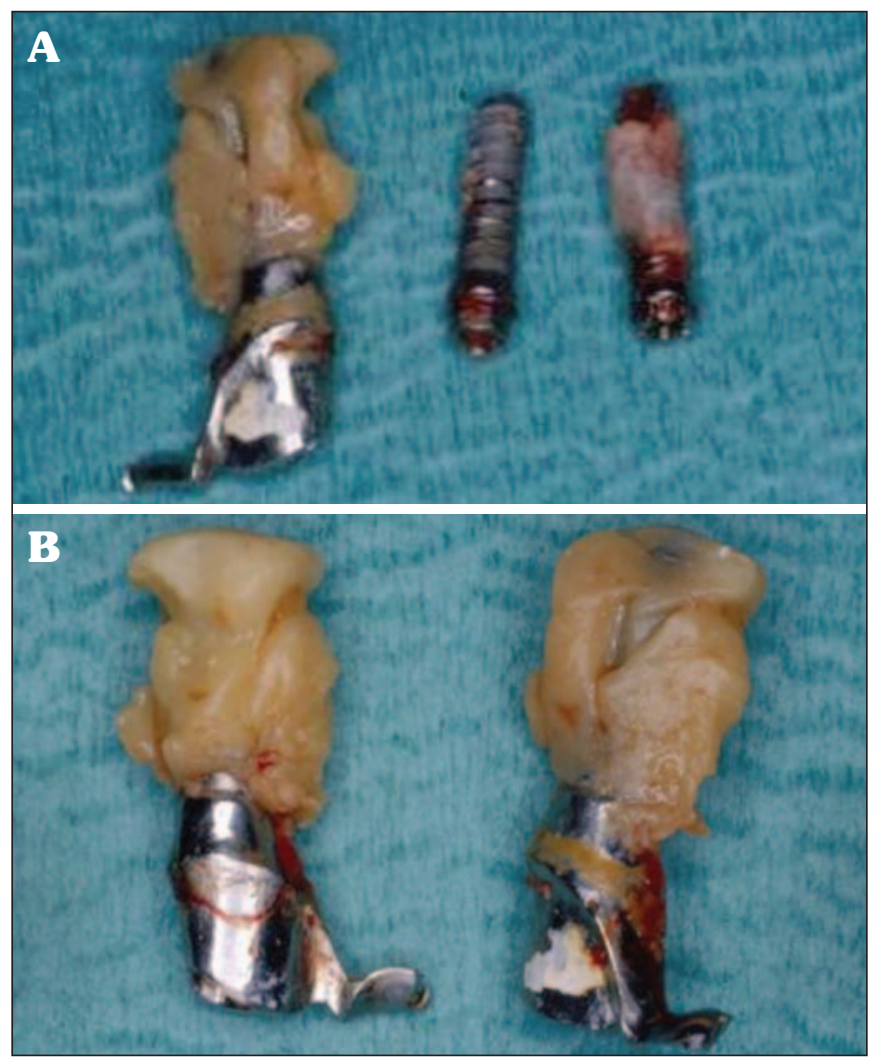

Fig. 6: Aspecto de los tres implantes una vez extraídos. (A) El implante en posición 1.2 aparece recubierto de hueso tras su extracción con trefina. La superficie del implante en posición 1.3, retirado con relativa facilidad, está limpia. (B) Detalle del implante situado en posición 1.6 y del material de aspecto resinoso adherido a toda su superficie.

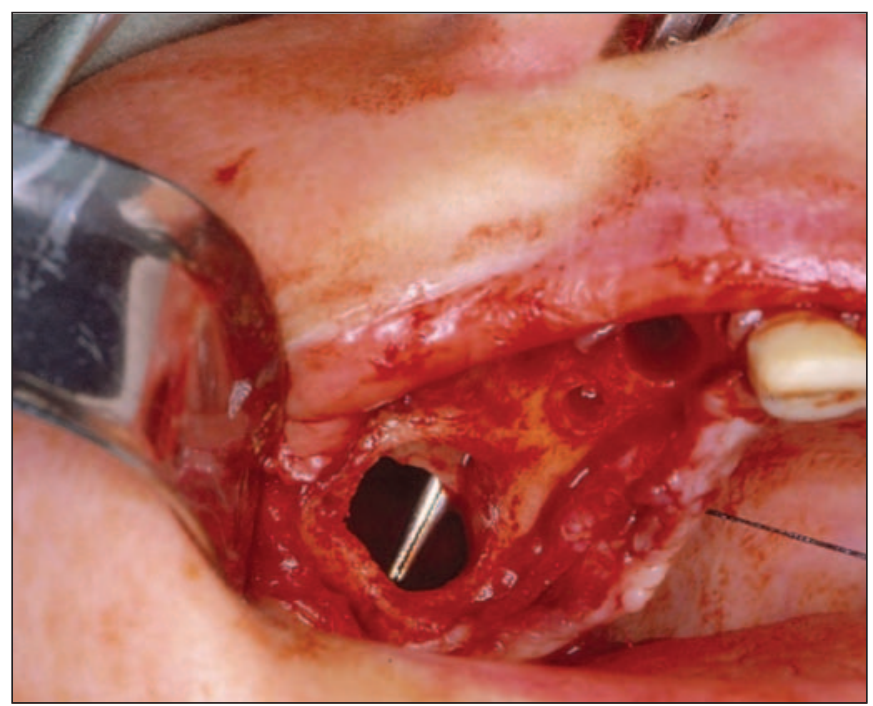

Fig. 7: Magnitud del defecto creado en el reborde alveolar tras retirar el implante en posición 1.6. Realización de una antrostomía nasal para facilitar el drenaje sinusal. un tubo de drenaje para favorecer la correcta aireación y drenaje del seno maxilar.

Para reconstruir los defectos óseos fue necesario utilizar hueso autólogo, por lo que se abrió un segundo campo quirúrgico en el mentón. Se infiltró anestesia local en fondo de vestíbulo del sector intercanino inferior y se levantó un colgajo semilunar modificado obteniéndose hueso de este área con un rascador, para regenerar el defecto producido al retirar los dos primeros implantes (1.2 y 1.3). La pérdida de sustancia era tal en el tercero (1.6) que no existía soporte que admitiera material alguno. Se utilizó una membrana de colágeno BioGide $囚$ (Geistlich Biomaterials, Wolhusen, Suiza) para sellar la comunicación oroantral y se preparó un colgajo pediculado de la bola adiposa de Bichat para conseguir un cierre primario de la herida, debido a que la destrucción tisular existente no permitía afrontar los tejidos borde a borde. A través de una incisión vertical de $1 \mathrm{~cm}$ de longitud sobre el periostio del proceso cigomático, se expuso la bola adiposa de Bichat, que fue disecada y traccionada delicadamente para desplazarla hacia el defecto óseo. Posteriormente se suturó a la mucosa palatina sin tensión utilizando una sutura reabsorbible de $4 / 0$ (Vicryl@, Johnson \& Johnson, New Brunswick, EE.UU.). Finalmente el colgajo mucoperióstico fue reposicionado sin tensión en la zona vestibular, dejando la parte alveolar del injerto adiposo expuesto a la cavidad bucal sin necesidad de colocar un apósito o cemento quirúrgico (Fig. 8). La medicación postoperatoria prescrita fue: Augmentine $®$ (GlaxoSmithKline, Madrid, España) 875/125 mg: 1 cápsula cada 8 horas durante 15 días, Enantyum $®$ (Laboratorios Menarini, Badalona, España) $25 \mathrm{mg}$ : 1 comprimido cada 8 horas durante 7 días, y Clorhexidina gel (Lacer, Cerdanyola del Vallés, España), aplicaciones tópicas sobre la herida 2 veces al día durante 15 días. En cuanto a las instrucciones postoperatorias se hizo especial hincapié en que evitase situaciones que aumentasen la presión intranasal como estornudar u otras maniobras de Valsalva, ingesta de alimentos líquidos o triturados en la semana siguiente a la intervención quirúrgica, y hacer vahos con manzanilla todas las noches a partir del tercer día.

El curso postoperatorio transcurrió sin complicaciones. Se programaron controles postoperatorios al día siguiente, a los 3 y a los 7 días, retirándose el drenaje en esta última visita. La paciente no refería molestias, habiendo prescindido casi por completo de la medicación analgésica. La herida presentaba buen estado, apreciándose una pequeña zona eritematosa en el fon- 

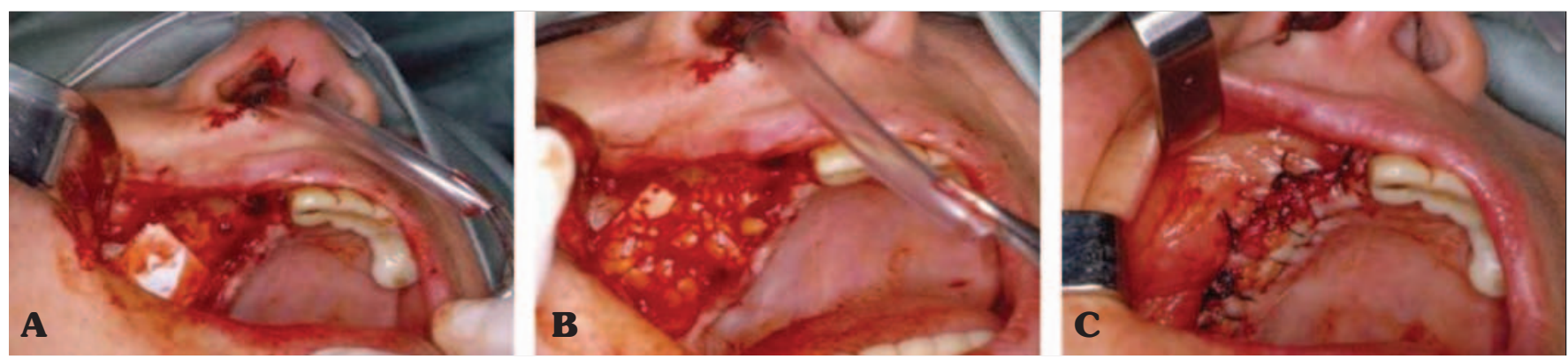

Fig. 8: Cierre del defecto creado. (A) Cierre de la comunicación bucosinusal producida a nivel del implante en posición 1.6 utilizando una membrana reabsorbible. Colocación del tubo de drenaje desde la fosa nasal derecha. (B) Inmovilización del colgajo pediculado de la bola adiposa de Bichat a la fibromucosa palatina mediante sutura reabsorbible. (C) Colgajo de la mucosa vestibular reposicionado: Se aprecian zonas donde el tejido adiposo queda expuesto a la cavidad bucal.

do del vestíbulo y un cambio en la coloración y aspecto de la parte del injerto adiposo que había quedado expuesta. Recomendamos extremar la higiene bucodentaria. Los puntos de sutura se retiraron a los 15 días: el cierre de la herida en este momento era completo (Fig. 9). Los resultados del análisis histológico confirmaron una mucosa sinusal hipertrófica, con marcado edema y denso infiltrado inflamatorio de tipo crónico (Fig. 10). Se hicieron controles postoperatorios periódicos e interconsultas con su otorrinolaringólogo para monitorizar el caso. A los seis meses de la intervención quirúrgica toda la sintomatología había desaparecido por completo y no presentaba tampoco hallazgos radiológicos en las ortopantomografías de control posteriores que evidenciasen patología (Fig. 11).

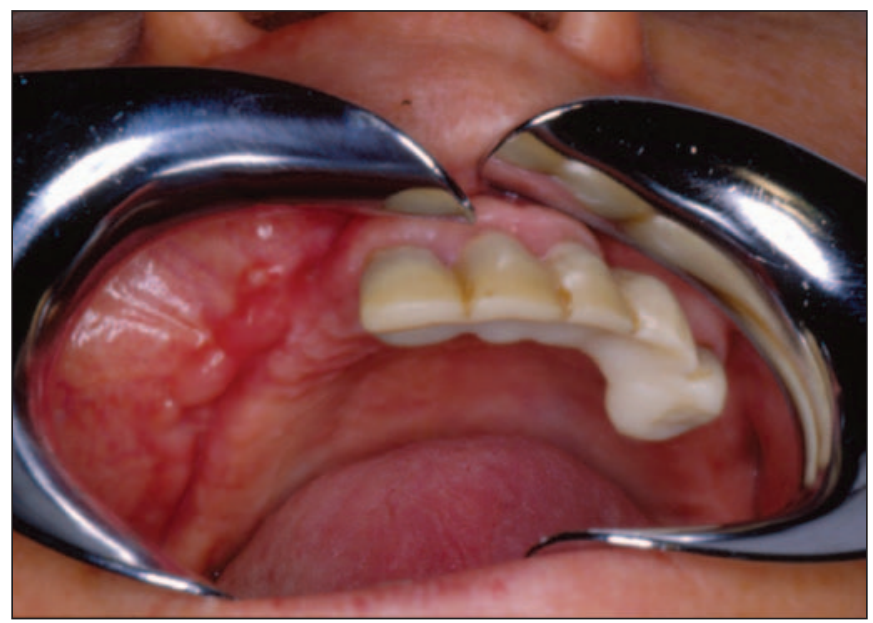

Fig. 9: Evolución en el postoperatorio: Aspecto a los quince días de la intervención quirúrgica tras retirar los puntos de sutura. Se observa área eritematosa residual remanente pero el tejido adiposo ha sufrido ya una metaplasia epitelial.

\section{DISCUSIÓN}

Una comunicación bucosinusal es una condición patológica que se caracteriza por la existencia de una solución de continuidad entre la cavidad bucal y el seno maxilar (3). Su etiología es variada y puede producirse a causa de la extracción tanto convencional como quirúrgica de los dientes posteriores del maxilar superior, por la penetración de raíces o dientes completos dentro del seno maxilar debido a maniobras intempestivas durante la exodoncia, en la cirugía periapical de molares y premolares superiores y, como es nuestro caso, tras la colocación de implantes. Otras entidades que pueden conducir a esta patología son quistes sinusales u odontogénicos del maxilar superior, neoplasias, osteomielitis, fisuras alveolopalatinas, traumatismos y más raramente enfermedades generales como la sífilis, la lepra y el SIDA (12).

Para confirmar su diagnóstico, a una exhaustiva anamnesis y exploración clínica debe unirse un estudio radiológico adecuado. Las radiografías intrabucales (periapicales y oclusales) pueden utilizarse, si bien son preferibles las extrabucales: la ortopantomografía es muy útil en el diagnóstico de la patología sinusal, sin embargo, otras proyecciones como la de Waters o Blondeau, y la incidencia de Hirtz ofrecen mejores imágenes del seno maxilar. No obstante, la técnica de elección en la actualidad es la tomografía computadorizada, con la que obtenemos una imagen nítida y concreta del grado de afectación sinusal y la extensión del defecto creado. Resulta de especial utilidad en comunicaciones producidas por traumatismos y en las ocasionadas tras grandes resecciones por tumores tanto benignos como malignos, para planificar la reconstrucción y cierre de los tejidos (13). 


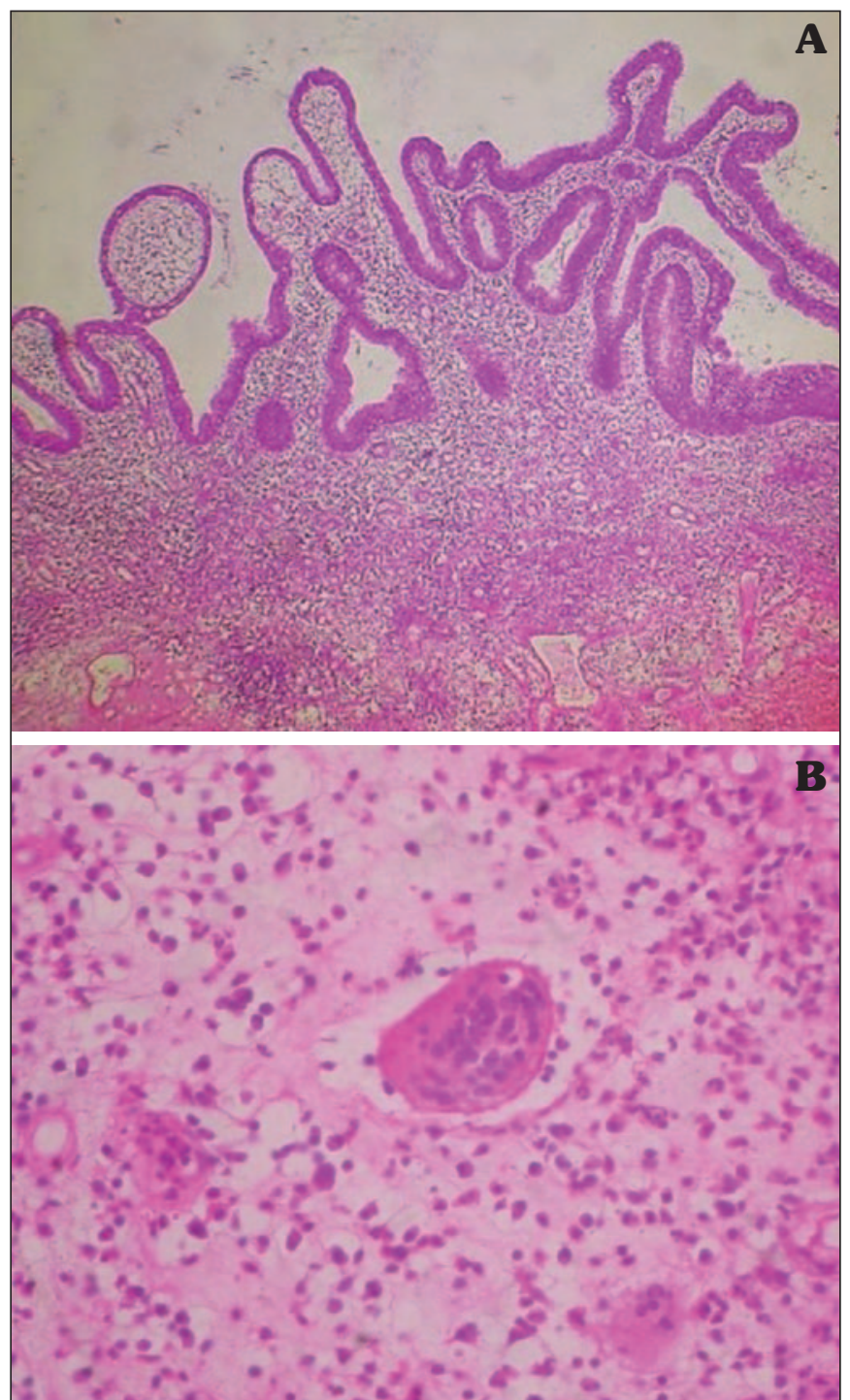

Fig. 10: Imágenes de la preparación histológica de las muestras obtenidas de la mucosa sinusal enferma (Hematoxilina-Eosina). (A) Mucosa con epitelio superficial hiperplásico y estroma edematoso. Proyecciones epiteliales de aspecto polipoide. (B) Infiltrado inflamatorio con predominio de células plasmáticas y presencia de eosinófilos. Proliferación de vasos capilares. Aparición ocasional de células gigantes multinucleadas.

La incidencia de sinusitis maxilar odontogénica, según los estudios consultados, se estima que ronda el 10$12 \%$ (14). Thevoz y cols. (15), tras una revisión retrospectiva de 197 pacientes con sinusitis maxilar tratados en su Departamento entre 1991 y 1999, cifran los casos de origen dentario en un $9 \%$. En 9 de los cuales $(5 \%)$ se identificaron en el interior del seno, como factor causal claro, distintos cuerpos extraños relacionados con algún tratamiento dental previo. La presencia
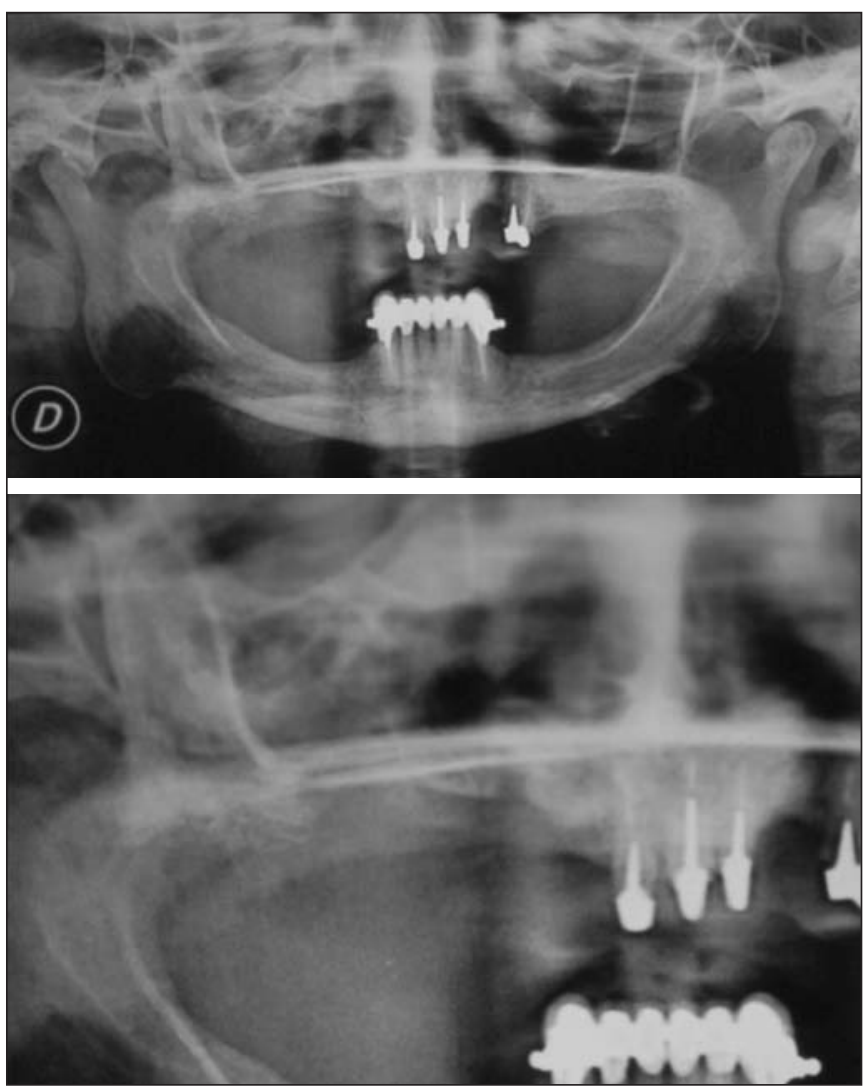

Fig. 11: Control radiológico transcurridos tres meses de la intervención quirúrgica. (A) Visión general de la ortopantomografía. (B) Detalle del primer cuadrante a mayor aumento. Se observa opacificación parcial remanente del seno maxilar derecho.

de distintos objetos y sustancias desplazadas al seno maxilar, como factores irritantes desencadenantes de sinusitis maxilar, está ampliamente registrada: desde amalgama $(16,17)$, gutapercha $(18,19)$, remanentes de tubos de plástico de drenajes previos (20) e incluso agujas de sutura (21). Debido a que estos cuerpos extraños pueden desplazarse por el interior del seno maxilar y fijarse a su pared posterior, con el consiguiente riesgo de extensión intracraneal del proceso infeccioso, y con la dificultad añadida en su extracción del alto riesgo de hemorragia de esta área. El tratamiento de elección en todos los casos suele ser la extracción inmediata mediante un abordaje siguiendo la técnica de Caldwell-Luc, y el legrado de toda la mucosa adyacente enferma (22). Será prioritario realizar el análisis histológico de una muestra de la mucosa sinusal extirpada, así como un cultivo, ya que es un hecho frecuente el hallazgo de cepas de Actinomyces y Aspergillus, y la resolución total del proceso requerirá tratamiento antimicrobiano específico durante al 
menos de 2 a 4 semanas posteriores a la intervención quirúrgica (17).

Existen pocos casos descritos en la literatura de migración de implantes dentales al interior de los senos maxilares (23-27). Las teorías propuestas en torno a las causas de este desplazamiento son múltiples: una inadecuada preparación quirúrgica del lecho implantario, dejando el implante sin la estabilidad primaria deseable; una periimplantitis que destruye el hueso circundante al implante comunicando de forma directa la cavidad bucal con el seno maxilar o incluso una mala distribución oclusal de las fuerzas en la prótesis definitiva, con la consiguiente pérdida ósea periimplantaria. Circunstancias todas ellas que, agravadas por la poca consistencia del hueso maxilar superior, explican la pérdida de anclaje implantario y su posterior desplazamiento (2). El implante actuará como un cuerpo extraño dentro del seno maxilar produciendo una infección de tipo agudo o crónico. La mayoría de autores $(2,28)$ coinciden en que debe tratarse como un cuerpo extraño y se debe extraer sin esperar a que produzca sintomatología, aprovechando esta intervención quirúrgica si el paciente no presenta infección sinusal activa, para aplicar técnicas de regeneración ósea como la elevación de seno maxilar o el aumento de cresta alveolar, que resultarán necesarias posteriormente para rehabilitar nuevamente y de forma definitiva este sector. En el caso de que en ese momento el paciente presente una reagudización del cuadro sinusal deberá esperarse a que remitan los síntomas clínicos y los signos radiológicos por completo, para implementar estos procedimientos en un segundo tiempo quirúrgico. Actualmente son ya muchos los autores (2931) que proponen, como alternativa al abordaje tradicional, la endoscopia nasal, una técnica simple y mínimamente invasiva. Puede utilizarse esta vía como coadyuvante, buscando el acceso intraoral convencional, o simplemente agrandar el ostium sinusal y efectuar la extracción del implante a través del mismo.

Las perforaciones de pequeño tamaño de la membrana de Schneider durante la preparación del lecho implantario no parecen estar relacionadas con el desarrollo de sinusitis postoperatoria en pacientes sanos, ni parecen afectar los resultados o el pronóstico a largo plazo de la rehabilitación protésica. Normalmente se cierran espontáneamente si no existe infección previa y se forma un coágulo estable (2); sin embargo, las perforaciones de gran tamaño deberán ser reparadas con adhesivo de fibrina o con una membrana de colágeno para evitar complicaciones. Ya en 1984,Brånemark y cols (32), tras realizar un estudio piloto en perros co- locando implantes que perforasen la base del seno maxilar y de las fosas nasales, y analizar los tejidos duros y blandos periimplantarios transcurrido un año de función en boca, no encontraron hallazgos radiológicos o histológicos que mostrasen ningún signo de reacción adversa: los implantes aparecían integrados en toda su superficie sin interposición de tejido fibroso, por lo que decidieron reproducirlo en humanos. Se colocaron un total de 139 implantes en 101 pacientes que, de manera similar a la descrita anteriormente, los porcentajes de éxito a los 5 y 10 años de seguimiento fueron de 96 y $88 \%$ respectivamente. Más recientemente, Adell y cols. (33) publicaron una serie de $371 \mathrm{pa}-$ cientes a quienes se había colocado implantes osteointegrados para su rehabilitación. Un total de 101 implantes colocados en el maxilar superior habían perforado el suelo del seno maxilar debido a que la altura del hueso alveolar era insuficiente y se buscaba su bicorticalización para ganar en estabilidad primaria, sin presentarse ningún caso de sinusitis en un seguimiento de hasta doce años.

En cuanto a los efectos de los implantes sobreextendidos al interior del seno maxilar, no parece existir evidencia científica en torno a que la porción del implante que perfora el suelo sinusal pueda evitar la recuperación espontánea de la laceración. En un estudio efectuado en perros de la raza mongrel a los que se les colocaron implantes dentales con su extremo apical parcialmente introducido en el seno maxilar, Jung y cols. (34) registraron que, en los casos en los que la porción penetrante del implante era menor o igual a $4 \mathrm{~mm}$, se cubría de nuevo por membrana sinusal sana que cicatrizaba por encima del mismo. Sin embargo, cuando esta longitud era superior a este valor, el implante quedaba expuesto a la cavidad sinusal con inserción directa de la membrana antral al titanio en la base del mismo, sin observarse cambios inflamatorios ni patológicos, y siendo la osteointegración de los implantes completa en todos los casos. En un estudio retrospectivo efectuado por el mismo equipo para intentar contrastar estas conclusiones con lo que ocurre en humanos (35), se identificaron 9 casos con un total de 23 implantes que perforaban el suelo del seno maxilar. Revisando de forma más meticulosa estos historiales se registró un engrosamiento asintomático de la mucosa en casi la mitad de los mismos, por lo que concluyen que no se puede descartar que en seguimientos a largo plazo no desencadene una sinusitis. Timmenga y cols. (36) han constatado que la sinusitis posterior a una elevación de seno sólo se desarrolla en individuos susceptibles, es decir, con una predisposición a esta patología, por lo que se podría 
extrapolar que esta hipertrofia mucosa, susceptible de afectar el complejo osteo-meatal y provocar patología subsecuente a su obstrucción, se produciría solamente en estos pacientes.

La creciente popularidad de los implantes en Odontología, junto al interés económico que estos suscitan, han hecho que una multitud de profesionales sin experiencia e insuficientemente preparados para solventar las complicaciones que pueden ocasionar, se lancen a colocarlos sin dubitaciones. Dentro de la práctica de cualquier especialidad se producen errores, sin embargo, el caso que presentamos es un claro ejemplo de iatrogenia injustificable por muchos motivos: Un estudio preoperatorio adecuado del caso debiera haber conducido a una elevación de seno previa a la colocación de implantes. Por otra parte, la gran comunicación bucosinusal producida intraoperatoriamente en la preparación del lecho del tercer implante debiera haber hecho desistir al operador. Sin embargo, intentó ocultar el problema mediante la fijación del implante "in situ" con resina, material altamente irritante que, en contacto directo con la mucosa sinusal, agravó la sinusitis maxilar y la comunicación bucosinusal generadas inicialmente.

\section{AGRADECIMIENTOS}

Este estudio ha sido realizado por el grupo de investigación consolidado "Patología y Terapéutica Odontológica y Maxilofacial" del Instituto IDIBELL con el soporte económico del convenio docente-asistencial de Cirugía Bucal entre la Universidad de Barcelona, el Consorci Sanitari Integral y el Servei Català de la Salut de la Generalitat de Catalunya.

\section{BIBLIOGRAFÍA}

1. Sánchez Garcés MA, Herrero M, Fuster MA, Ferrús E, Gay Escoda C. Revisión bibliográfica de implantología bucofacial del año 2005. $1^{\text {a }}$ Parte. Arch Odontoestomatol 2007;23:45-62.

2. Raghoebar GM, Vissink A. Treatment for an endosseous implant migrated into the maxillary sinus not causing maxillary sinusitis: Case report. Int J Oral Maxillofac Implants 2003;18:745-9.

3. Gay Escoda C. Comunicaciones bucosinusales. En: Gay Escoda C, Berini Aytés L, (eds). Cirugía Bucal. Madrid: Ergon; 1999.
4. Egyedi P. Utilization of the buccal fat pad for closure of oroantral and/or oronasal communications. J Maxillofac Surg 1977;5:341-4.

5. Scott P, Fabbroni G, Mitchell DA. The buccal fat pad in the closure of oro-antral communications: An illustrated guide. Dent Update 2004;31:363-6.

6. Colella G, Tartaro G, Giudice A. The buccal fat pad in oral reconstruction. Br J Plast Surg 2004;57:326-9.

7. Tideman $H$, Bosanquet $A$, Scott J. Use of the buccal fat pad as a pedicled graft. J Oral Maxillofac Surg 1986;44:435-40.

8. Samman N, Cheung LK, Tideman H. The buccal fat pad in oral reconstruction. Int J Oral Maxillofac Surg 1993; 22:2-6.

9. Abuabara A, Cortez AL, Passeri LA, de Moraes M, Moreira RW. Evaluation of different treatments for oroantral/oronasal communications: Experience of 112 cases. Int J Oral Maxillofac Surg 2006;35:155-8.

10. Stuzin JM, Wagstrom L, Kawamoto HK. The anatomy and clinical applications of the buccal fat pad. Plast Reconstr Surg 1990;85:29-37.

11. Baumann A, Ewers R. Application of the buccal fat pad in oral reconstruction. J Oral Maxillofac Surg 2000;58: 389-93.

12. Sada JM. Comunicaciones bucosinusales y buconasales. En: Donado M, (ed). Cirugía Bucal. Patología y técnica. $3^{\mathrm{a}}$ ed. Madrid: Masson; 2005.

13. Vericat Queralt A, Berini Aytés L, Gay Escoda C. Tratamiento quirúrgico de las comunicaciones bucosinusales. Rev Vasca Odontoestomatol 2000;10:10-23.

14. Brook I. Sinusitis of odontogenic origin. Otolaryngol Head Neck Surg 2006;135:349-55.

15. Thevoz F, Arza A, Jaques B. Dental foreign body sinusitis. Schweiz Med Wochenschr 2000;125:30-34.

16. Macan D, Cabov T, Kobler P, Bumber Z. Inflammatory reaction to foreign body (amalgam) in the maxillary sinus misdiagnosed as an ethmoid tumor. Dentomaxillofac Radiol 2006;35:303-6.

17. Selmani Z, Ashammakhi N. Surgical treatment of amalgam fillings causing iatrogenic sinusitis. J Craniofac Surg 2006; 17:363-5. 
18. Ishikawa M, Mizuno T, Yamazaki Y, Satoh T, Notani K, Fukuda H. Migration of gutta-percha point from a root canal into the ethmoid sinus. Br J Oral Maxillofac Surg 2004;42:58-60.

19. Yamaguchi K, Matsunaga T, Hayashi Y. Gross extrusion of endodontic obturation materials into the maxillary sinus: A case report. Oral Surg Oral Med Oral Pathol Oral Radiol Endod 2007;104:131-4.

20. Hasegawa J, Watanabe $\mathrm{K}$, Kunitomo M, Yamauchi $\mathrm{Y}$, Kurosaki S, Higa I, et al. Foreign body in the maxillary sinus-possible plastic tube: A case report. Auris Nasus Larynx 2003;30:299-301.

21. Fan VT, Korvi S. Sewing needle in the maxillary antrum. J Oral Maxillofac Surg 2002;60:334-6.

22. Krause HR, Rustemeyer J, Grunert RR. Foreign body in paranasal sinuses. Mund Kiefer Gesichtschir 2002;6:40-4.

23. Cedeño R, Costa S. Migración de implantes al seno maxilar. A propósito de un caso clínico. Rev Oper Dent Endod 2006;5:50.

24. Haben CM, Balys R, Frenkiel S. Dental implant migration into the ethmoid sinus. J Otolaryngol 2003;32:342-4.

25. Gallego Medina I, Sánchez Garcés M, Berini Aytés L, Gay Escoda C. Desplazamiento de un implante dental dentro del seno maxilar durante la segunda fase quirúrgica. Av Periodon Implantol 2002;14:81-8.

26. Iida S, Tanaka N, Kogo M, Matsuya T. Migration of a dental implant into the maxillary sinus. A case report. Int J Oral Maxillofac Surg 2000;29:358-9.

27. Gümru O, Kocak H. Acute maxillary sinusitis from a dental implant: An unusual case report.J Marmara Univ Dent Fac 1996;2:551-3.

28. Galindo P, Sánchez-Fernández E, Ávila G, Cutando A, Fernández JE. Migration of implants into the maxillary sinus: Two clinical cases. Int J Oral Maxillofac Implants 2005;20:291-5.

29. Kitamura A. Removal of a migrated dental implant from a maxillary sinus by transnasal endoscopy. Br J Oral Maxillofac Surg 2007;45:410-1.
30. Friedlich J, Rittenberg BN. Endoscopically assisted Caldwell-Luc procedure for removal of a foreign body from the maxillary sinus. J Can Dent Assoc 2005;71: 200-1.

31. Kim JW, Lee CH, Kwon TK, Kim DK. Endoscopic removal of a dental implant through a middle meatal antrostomy. Br J Oral Maxillofac Surg 2007;45:408-9.

32. Branemark PI, Adell R, Albrektsson T, Lekholm U, Lindstrom J, Rockler B. An experimental and clinical study of osseointegrated implants penetrating the nasal cavity and maxillary sinus. J Oral Maxillofac Surg 1984;42:497-505.

33. Adell R, Eriksson B, Lekholm U, Branemark PI, Jemt T. Long-term follow-up study of osseointegrated implants in the treatment of totally edentulous jaws. Int J Oral Maxillofac Implants 1990;5:347-59.

34. Jung JH, Choi BH, Jeong SM, Li J, Lee SH, Lee HJ. A retrospective study of the effects on sinus complications of exposing dental implants to the maxillary sinus cavity. Oral Surg Oral Med Oral Pathol Oral Radiol Endod 2007; 103:623-5.

35. Jung JH, Choi BH, Zhu SJ, Lee SH, Huh JY, You TM, et al. The effects of exposing dental implants to the maxillary sinus cavity on sinus complications. Oral Surg Oral Med Oral Pathol Oral Radiol Endod 2006;102: 602-5.

36. Timmenga NM, Raghoebar GM, Boering G,Weissenbruch R. Maxillary sinus function after sinus lifts for the insertion of dental implants. J Oral Maxillofac Surg 1997; 55:936-9.

\section{CORRESPONDENCIA}

\section{Dr. Cosme Gay Escoda}

Centro Médico Teknon

Instituto de Investigación Biomédica IDIBELL.

Vilana, 12

08022 Barcelona

e-mail: cgay@ub.edu

http:// www.gayescoda.com 